\title{
Mapping Emotions Through Time: How Affective Trajectories Inform the Language of Emotion
}

\author{
Tabitha Kirkland and William A. Cunningham \\ The Ohio State University
}

\begin{abstract}
The words used to describe emotions can provide insight into the basic processes that contribute to emotional experience. We propose that emotions arise partly from interacting evaluations of one's current affective state, previous affective state, predictions for how these may change in the future, and the experienced outcomes following these predictions. These states can be represented and inferred from neural systems that encode shifts in outcomes and make predictions. In two studies, we demonstrate that emotion labels are reliably differentiated from one another using only simple cues about these affective trajectories through time. For example, when a worse-than-expected outcome follows the prediction that something good will happen, that situation is labeled as causing anger, whereas when a worse-thanexpected outcome follows the prediction that something bad will happen, that situation is labeled as causing sadness. Emotion categories are more differentiated when participants are required to think categorically than when participants have the option to consider multiple emotions and degrees of emotions. This work indicates that information about affective movement through time and changes in affective trajectory may be a fundamental aspect of emotion categories. Future studies of emotion must account for the dynamic way that we absorb and process information.
\end{abstract}

Keywords: emotion, affect, categorization, affective trajectory, iterative reprocessing

The words we use to describe, label, and categorize emotions have provided insight into the nature of affective processing (Clore, Ortony, \& Foss, 1987; Johnson-Laird \& Oatley, 1989; Storm \& Storm, 1987). Since the early days of psychology, the dominant view has been that these linguistic categories correspond in a one-to-one relationship with underlying discrete emotional states (Ekman \& Friesen, 1971). For example, the word fear exists because it represents a nondivisible biological category of a fear or threat process. However, newer work suggests that emotions are not fundamental or elemental units of the mind but instead are the emergent products of simpler, more versatile components. Just as a loaf of bread does not reveal the ingredients that constitute it, most experienced psychological states do not reveal their underlying components (Barrett, 2009). These basic psychological ingredients-or psychological primitives (Ortony \& Turner, 1990) — can combine in various ways to produce a variety of mental experiences, including emotion. This is the essence of an approach termed psychological constructivism. We label the subjective sense of impending doom fear, but this is not because a fear center has been activated. Although this insight has recently gained traction in the emotion literature, it remains unclear what these ingredients are and how they interact to create the subjective sense of emotion and the emotion categories used by nearly all cultures (Barrett, 2009; Cunningham \& Van Bavel, 2009).

This article was published Online First July 25, 2011.

Tabitha Kirkland and William A. Cunningham, Department of Psychology, The Ohio State University.

Correspondence concerning this article should be addressed to Tabitha Kirkland or William A. Cunningham, Department of Psychology, The Ohio State University, 1835 Neil Avenue, Columbus, OH 43210. E-mail: kirkland.37@osu.edu or cunningham.417@osu.edu
Based on the iterative reprocessing model of evaluation (Cunningham \& Zelazo, 2007; Zelazo \& Cunningham, 2007), we now have a psychological constructivist account of the cognitive and neural bases of affective processes, the affective trajectories hypothesis (Cunningham \& Van Bavel, 2009; Cunningham \& Zelazo, 2009; Kirkland \& Cunningham, in press), which suggests that we must account for dynamically shifting cognitive processes to fully understand the psychological construction of subjective emotional states. It is not sufficient to know that someone is feeling negative in a more or less intense way; one also needs to understand the temporal shifts in affect that got the person to his current state. According to this account, emotions arise in part from the interaction of the evaluations of one's current state, predictions for the future, and the outcomes that one experiences after these predictions (Cunningham \& Van Bavel, 2009; Cunningham \& Zelazo, 2009; Kirkland \& Cunningham, in press). These evaluative processes interact to create a gestalt emotional impression. Differentially valenced combinations of these factors lead to qualitatively distinct affective states, which are then categorized and elaborated upon.

In contrast to social constructivist models, which propose that emotion categories are arbitrary, the affective trajectories hypothesis suggests that the labels that are used reflect underlying patterns of affective processing. Specifically, we suggest that the labels represent, at least in part, our affective trajectories through time. These trajectories provide critical cues about how we reached our current affective state and what we predict for the future. For example, a mildly positive affective state can be construed as pleasant or aversive, depending on whether it follows a worse or better state, respectively. Consistent with this idea, temporal focus-attention to the present or the future-is associated with 
separate emotion labels (Ortony, Clore, \& Collins, 1988; Roseman, 1984). According to this view, a predicted negative state may be labeled fear, whereas a current negative state may be labeled sadness. Differentiating between these categories is important because they provide information as to where in the system the change has occurred. A negative event that can still be avoided may lead to a different set of behavioral options than one that has already occurred (Lazarus, 1982). If these labels represent underlying psychological states, it stands to reason that people should have an intuitive understanding of the association between emotion labels and their underlying topography. In two studies, we explore the idea that, given varying combinations of current state, prediction, and outcome, people should be able to reliably differentiate the emotional states that follow. This research builds on previous work yet takes a slightly different approach. All major emotion theories thus far have focused on trying to identify the basic ingredients of emotion; the present approach makes the unique prediction that people will be able to distinguish emotion words from one another only on the basis of simple cues about a person's affective trajectory through time.

\section{Emotions as Constructed}

Although we readily recognize and label emotions in ourselves and others, our extensive vocabulary for describing specific emotional experience (e.g., Shaver, Schwartz, Kirson, \& O'Connor, 1987) does not necessarily reflect the underlying or latent structure of emotion. Some researchers have suggested that, rather than existing as discrete and immobile entities, emotions are constructed from more elemental processing units. An analogy can be made to the way that our visual system operates to identify objects. Research in visual perception has demonstrated that our visual systems are calibrated to recognize basic perceptual units such as squares, triangles, and circles; recognition of complex objects is organized in terms of these simple units (Biederman, 1987). For example, one configuration of a cylinder and a curved line produces the perception of a coffee mug; in a different configuration, the same cylinder and curved line may be perceived as a bucket. However, the final percept is not of the elements but of the gestalt impression. Similarly, we may also be calibrated to respond to basic mental units with various forms of cognition or affect, depending on the configuration. In the right configuration, these units provide the subjective impression of basic emotions. Thus, the question has transformed from, "What are the basic emotions?" to "If emotions are composed of simpler ingredients, what are these ingredients, and how are they combined?" That is, what basic processes go into the experience of an emotion, and what types of interactions occur among these processes? An early approach to this question involved conducting factor analysis on labels of emotion (e.g., Schlosberg, 1952). This approach used model-free cluster and factor analyses to uncover the latent structure of words, asserting that the language that we use to describe emotions can give clues as to our intuitive sense of its underlying structure. Typically, a circumplex emerges from these analyses consisting of two dimensions along which affect can vary, such as valence and arousal (Plutchik, 1962; Russell, 1980, 2003; Watson \& Tellegen, 1985; Yik, Russell, \& Barrett, 1999). This work was quite generative and eventually led to a more general conceptualization of the structure of affect and other psychological states, termed psychological constructivism.

A psychological constructivist approach suggests that we have a limited number of basic mental ingredients that can be combined in various ways to produce a number of different experiences, including emotions, thoughts, memories, and so forth. These fundamental components of our mental life are flexible and multifunctional. What these ingredients are, specifically, is debated; researchers have varyingly pointed to the roles of the physical context (e.g., Carroll \& Russell, 1996; Schachter \& Singer, 1962), psychological context (e.g., Lindquist \& Barrett, 2008; Lindquist, Barrett, Bliss-Moreau, \& Russell, 2006), and cultural context (Barrett, Mesquita \& Gendron, in press) as factors that may influence the construction of emotion. For example, core affect, categorization processes, and attentional mechanisms have been nominated as constituting a partial list of these ingredients (Barrett, 2009). Although the term psychological constructivism is relatively new (Barrett, 2009), an analysis of prominent theories over the past half century suggests that this approach has implicitly informed many models of emotion, whether those ingredients are valence and arousal, cognitive appraisals, or other elements (Gendron \& Barrett, 2009).

The iterative reprocessing model and the affective trajectories hypothesis offer a complementary approach. On the basis of recent neuroscience research, the iterative reprocessing model reflects the dynamic nature of evaluation, highlighting the fact that we are constantly monitoring our situation and updating our representations of that situation as appropriate (Cunningham \& Zelazo, 2007). According to the iterative reprocessing model, evaluative states are constructed dynamically through a series of iterative neural loops occurring multiple times per second. These multiple mental systems serve as a way of tracking our affective trajectories through time. Incoming information can inform the system as it is compared with previous information, and the discrepancy between these two states is computed. This, in turn, informs interpretation of future updates. Thus, we are constantly situated at a time point in affective space comprising our past, our present, and what we predict for our future. The current affective state is constructed on the basis of the newest incoming information (what just happened, including comparisons to previous predictions) and the information that existed before (past events or feeling states), as well as any predictions being made about what may occur next (see also Carver \& Scheier, 1990, for a similar argument about goal pursuit). Our emotion categories are, thus, a way to label and differentiate the various affective trajectories we experience as we move continuously through time.

This framework is consistent with the commonly held view that emotions emerge from the interaction of cognitive processes with more basic affective cues (Barrett, 2006; Clore \& Huntsinger, 2009). Although the earliest incoming information may be simple cues of valence and comparison processes, at subsequent time points in processing the system can handle increasingly complex and subtle information, including cognitive appraisals, action tendencies (e.g., approach or avoid), social cues, and so forth. In this way, basic emotion categories can be emergent properties of more simple computations of valence in a hierarchical cognitive system. This approach allows for the generation of discrete emotional states while conforming to current beliefs about the cognitive and neural architecture of the human mind. 
Turning to the neuroscience literature can provide some additional support for affective processes as partially independent. Early work in affective neuroscience attempted to identify discrete modules corresponding to specific emotions. For example, some studies demonstrate that fear may be linked to the amygdala (Adolphs, 1999; Adolphs, Tranel, Damasio, \& Damasio, 1994; Bechara et al., 1995; see Phan, Wager, Taylor, \& Liberzon, 2002, for a meta-analysis), whereas disgust may be linked to the insula (Calder, Keane, Manes, Antoun, \& Young, 2000). Studies of depression and sadness (and, more recently, joy and excitement) found that these emotions may be associated with activation changes in medial frontal regions such as the cingulate and medial orbitofrontal cortex (Elliott \& Dolan, 2003; Mayberg et al., 1999). Furthermore, a recent meta-analysis reveals that anger may be particularly associated with activation in the lateral orbitofrontal cortex (Murphy, Nimmo-Smith, \& Lawrence, 2003).

However, finding activation within a given neural region does not allow for the reverse inference of knowing which emotion, if any, is occurring (Cacioppo \& Tassinary, 1990). For example, although inducing fear will most likely yield an amygdala response, amygdala activation is also found in inductions of other affective states, suggesting that the same brain regions may be used in multiple affective states. That is, the knowledge that fear leads to an amygdala response often results in the logically incorrect inference that amygdala response indicates fear activation. The brain is highly interconnected, and many different stimuli have been shown to be associated with amygdala activity, such as motivationally salient stimuli (Cunningham, Van Bavel, \& Johnsen, 2008), pleasant stimuli (Garavan, Pendergrass, Ross, Stein, \& Risinger, 2001; Hamann, Ely, Hoffman, \& Kilts, 2002), and novel stimuli (Schwartz et al., 2003; Wilson \& Rolls, 1993).

These findings imply that activation within brain areas associated with certain emotions does not necessarily indicate the experience of the associated emotion. In other words, the correspondence between the brain areas involved in an emotional experience and the emotions themselves may not exhibit a one-to-one mapping. Indeed, recent quantitative meta-analyses of the neuroimaging of emotion literature have failed to detect direct mapping between brain activation patterns and specific emotions, instead finding certain brain areas to be associated with some emotions in some studies and other emotions in other studies (Lindquist, Wager, Kober, Bliss-Moreau, \& Barrett, in press). Although a direct mapping between specific brain regions and specific emotions may not be tenable, this does not imply that the brain processes associated with emotion are interchangeable. As such, it is important to determine the processes that underlie affective responses and how they combine to generate emotional experience.

In one attempt to uncover the ingredients of emotion, Rolls (2000) proposed that our emotion categories stem from patterns of reward processing (see Kringelbach \& Rolls, 2004; O'Doherty, 2004; Schultz \& Dickinson, 2000). He proposed that the words that we use to understand emotional states arise from the processes that encode rewards and punishments; specifically, whether those rewards or punishments are delivered or not. For example, some types of emotions may arise depending on whether one receives rewards or punishments: Joy follows from receiving a reward, whereas fear follows from receiving a punishment. Other emotions may arise if an expected reward or punishment is omitted: Anger results from the omission of an expected reward and relief from the omission of an expected punishment. This model highlights the dynamic nature of emotional processes and explicitly considers the relationship between expectation and outcome.

However, despite the elegance of this model, it fails to fully represent our emotional topography. For example, we often label our affective state as fear not after the punishment has been received but at the time of generating the prediction of a negative outcome. Similarly, our feelings of happiness and sadness are not simply the result of our current circumstances but are in part determined by our previous affective states (Scherer, 1984; Smith \& Ellsworth, 1985). For example, changes in the rate of progress toward a goal can have affective implications: Contrasting past negative feelings with current positive feelings may lead to a "rush of exhilaration," whereas the reverse contrast may lead to a "sinking feeling" (Carver \& Scheier, 1990, p. 24). When assigning emotion labels to these feelings, we may label our mildly positive affective state as happy if we have been feeling relatively bad in the past or as sad if it follows a period of intense euphoria. Emotions are also partially determined by expectations of future affective states; when expectations for the future are positive, affect is positive, and when expectations are negative, affect is negative (Carver, 1979). Thus, we propose that our emotion labels may reflect not only rewards and punishments that are received or omitted but the full range of processes associated with reward processing. Specifically, we propose that emotion categories reflect, in part, the affective interpretations of our previous, predicted, and experienced outcomes, as well as the comparisons of these representations (see Cunningham \& Van Bavel, 2009; Kirkland \& Cunningham, in press). Thus, both the apparent correspondence between emotions and brain activity (according to conventional wisdom) and the vast heterogeneity of responses within these regions can potentially be resolved by considering these activations as representing "ingredients" of emotion rather than directly mapping to the emotion concepts themselves.

Combining the predictions developed from Rolls (2000) with the additional leverage that results from considering multiple temporal representations of affect, we propose a topography of emotion in which emotion categories represent different combinations of expected events and actual outcomes (see Tables 1, 2, and 3). For example, we predict that simply anticipating a negative event is sufficient for people to use the fear label, whereas anger typically requires a discrepancy between an anticipated and an experienced state. Furthermore, although we may label our emotion as joy or happiness when a positive event occurs, this is especially the case when the positive event occurs after a negative one. In other words, people amplify their sense of happiness by comparison with what once was. Thus, we suggest that whereas some emotions are highly related to predictions, others are outcome dependent, and still others require comparisons between prediction and outcome, reflecting a particular affective trajectory through time (Kirkland \& Cunningham, in press). Additional affective states (e.g., moods) may be reliant only on current information without necessitating comparisons. It is important to note that these comparisons do not require any contextual details or semantic appraisals. Whereas a cognitive appraisal-focused approach (e.g., Arnold, 1960; Lazarus, 1984; Scherer, 1993) predicts that emotion requires some degree of evaluation regarding the situation, likelihood of a particular outcome, goal consistency, and so forth, our model suggests that emotion labels (indeed, perhaps emotions themselves) can arise 
Table 1

Theoretical Predictions, Preoutcome

\begin{tabular}{|c|c|c|c|c|c|c|c|c|c|c|}
\hline Current & Prediction & Fear & Hope & Joy & Sad & Anger & Surprise & Content & Disgust & None \\
\hline $\mathrm{Bad}$ & $\mathrm{Bad}$ & 1 & & & & & & & & \\
\hline Good & $\mathrm{Bad}$ & 1 & & & & & & & & \\
\hline $\mathrm{Bad}$ & Good & & 1 & & & & & & & \\
\hline Good & Good & & 1 & & & & & & & \\
\hline $\mathrm{Bad}$ & None & & & & 2 & & & & & \\
\hline Good & None & & & & & & & 1 & & \\
\hline
\end{tabular}

Note. Theoretical predictions for the emotions relevant to each affective trajectory before the outcome. $1=$ a central prediction for a given emotion with a particular temporal pattern; 2 = a secondary prediction. Fear and hope are primarily prediction related: Fear will emerge in response to a negative prediction, and hope will emerge in response to a positive prediction. Contentment/calm will emerge as a mood state, existing without trajectory. Disgust is included for consistency with other major theories, but as it operates within a different neural system compared with the rest of the emotions, we predicted no systematic effects for disgust.

with only information about affective states in time. Although we do not mean to imply that they are the only processes that may inform emotion, we propose, critically, that affective trajectories may be among the fundamental building blocks of emotional experience and that trajectory-related information alone may help to differentiate emotion categories.

The present goal is to provide initial evidence that people can reliably differentiate emotion labels in patterns of affective information across time. Our affective topography-the rich mental mapping of affective information that constructs our affective states from moment to moment-is varied and complex, and we may have an instinctive sense of how changes in information across time will affect our emotions. This is congruent with the factor-analytic approach to studying emotion, which asserts that words suggest an underlying structure (e.g., Schlosberg, 1952); however, our approach is hypothesis-based rather than exploratory. In two studies, we parametrically manipulated the valence and presence of information about current state, prediction, and outcome and observed the emotion label that people selected as being representative of what they would feel given that particular combination of events. Information about these time states was decontextualized, containing only simple valence information (e.g., "you predict something bad is about to happen"). We ex- pected that people would be able to reliably identify certain emotion words to fit each situation in the specific pattern described earlier.

\section{Study 1: Categories}

\section{Method}

Participants. Forty undergraduate students (15 female, 25 male; age range $18-29$, mean age $=20.1$ ) participated in exchange for course credit in an introductory psychology class.

Procedure. The study was scheduled in sessions ranging in size from 1-6 people. After being informed as to the nature of the study and after giving their consent to participate, participants used individual computers to complete the experiment. The study was conducted with MediaLab v2006 (Jarvis, 2006). After completion of the experiment, participants were fully debriefed and thanked for participation.

During the experiment, participants read 18 separate scenarios in which the valence of current state, prediction, and outcome were varied systematically in a $2 \times 3 \times 3$ design. Specifically, participants were asked to imagine themselves in each situation in which they were feeling good (or bad), and they predicted something

Table 2

Theoretical Predictions, Postoutcome

\begin{tabular}{|c|c|c|c|c|c|c|c|c|c|c|}
\hline Prediction & Outcome & Fear & Hope & Joy & Sad & Anger & Surprise & Content & Disgust & None \\
\hline $\mathrm{Bad}$ & As expected & & & & 1 & & & & & \\
\hline Good & As expected & & & 1 & & & & & & \\
\hline Nothing & As expected & & & & & & & 1 & & 1 \\
\hline $\mathrm{Bad}$ & Better & & & 1 & & & 2 & & & \\
\hline Good & Better & & & 1 & & & 2 & & & \\
\hline Nothing & Better & & & 1 & & & 2 & & & \\
\hline $\mathrm{Bad}$ & Worse & & & & 1 & & 2 & & & \\
\hline Good & Worse & & & & & 1 & 2 & & & \\
\hline Nothing & Worse & & & & 1 & & 2 & & & \\
\hline
\end{tabular}

Note. Theoretical predictions for the emotions relevant to each affective trajectory after the outcome. $1=$ a central prediction for a given emotion with a particular temporal pattern; 2 = a secondary prediction. Joy and sadness will result from changes in affective trajectory: Joy will occur in response to a positive change (or upward trajectory); sadness will occur in response to a negative change (downward trajectory). Anger will occur in response to an unexpected negative change in affective trajectory; that is, when one's negative outcomes differ from one's positive predictions. Surprise will also occur in response to an unexpected change in trajectory, but it need not be inherently positive or negative. As in Table 1, disgust is included for consistency with other major theories, but as it operates within a different neural system compared with the rest of the emotions, we predicted no systematic effects for disgust. 


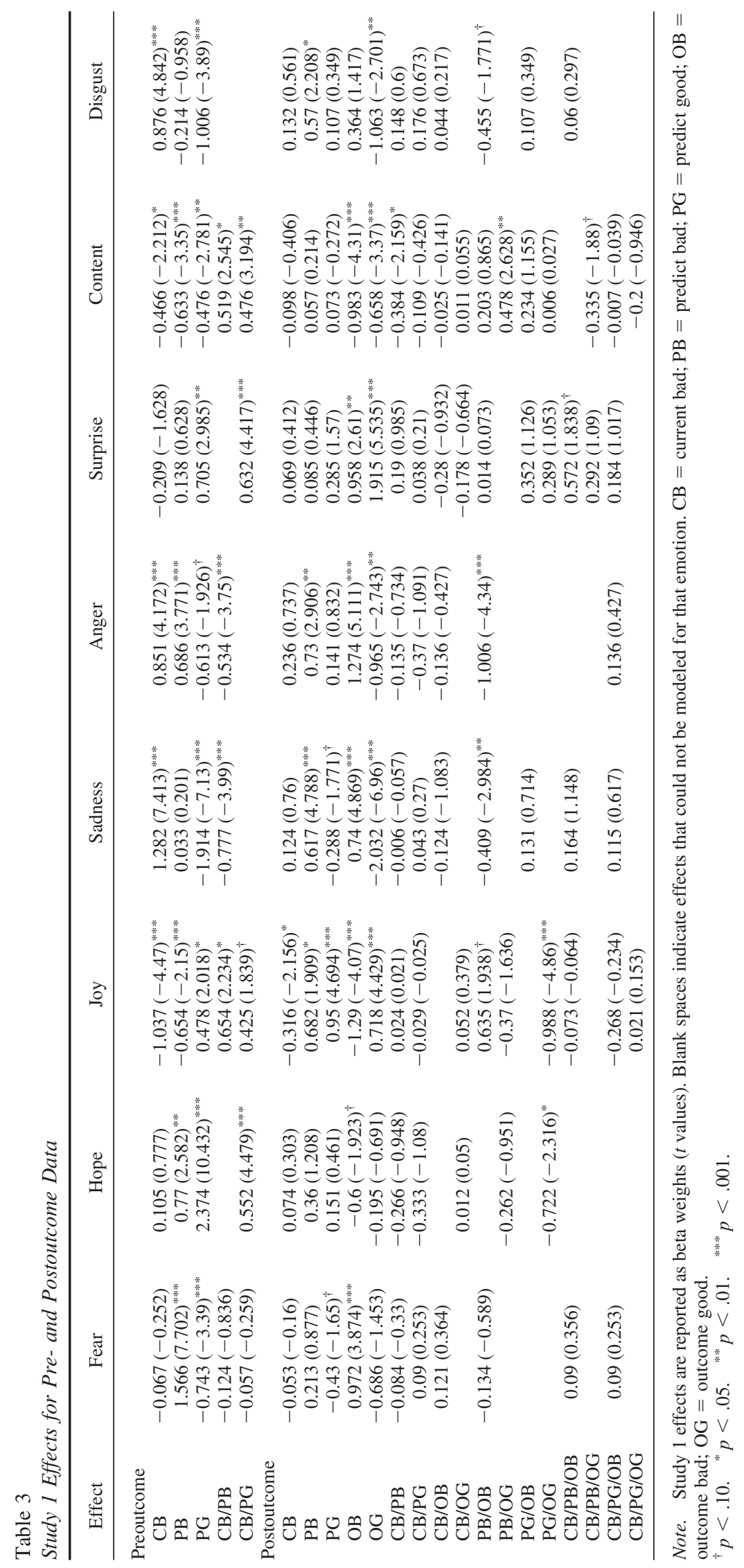


great would happen (or something terrible, or they had no prediction). Participants had as much time as they needed to read the screen $(M=2.841 \mathrm{~s})$ and pressed a button when they were ready to continue. Next, participants selected the emotion that was best represented in that scenario. They were given a representative list of emotions to choose from, including anger, contentment/calm, disgust, fear, hope, joy, sadness, surprise, and no emotional response. These particular emotion words were chosen with Ekman and Friesen's (1971) list of basic emotions, with some additional words from the Positive and Negative Affect Schedule (Watson \& Tellegen, 1985), to form a representative sample of emotions.

The second part of the scenario displayed the outcome, which was better than predicted, worse than predicted, or as predicted. For example, one scenario read, "You are feeling good. You predict something bad is going to happen ... . Instead of what you predicted, something far better happens." For outcomes to scenarios in which there had been no prediction, the positive outcome was phrased as "something great happens"; the negative outcome was phrased as "something terrible happens"; and the neutral outcome was phrased as "something ordinary happens." Thus, an affective trajectory was inherent in the wording of each time point. Again, participants were given as much time as needed to read the outcome ( $M=2.382 \mathrm{~s}$ ) and pressed a button to continue. Participants again selected the emotion that was best represented given the outcome, using the same list of emotions given in the preoutcome portion of the scenario. Thus, participants made two selections per scenario for a total of 36 emotion selections. The order of presentation was randomized across scenarios.

\section{Results}

We constructed a series of binomial hierarchical linear models (HLMs) predicting the selected emotion categories. The data were effect coded, and separate binomial outcome variables were created to indicate whether participants selected a particular emotion (e.g., anger $=1$, all others $=0$ ). These emotion variables were then predicted by our manipulated variables: current, prediction, and outcome. All interaction terms were modeled in addition to the main effects. Because we had separate theoretical predictions for the emotions that would be labeled at prediction and those after the outcome, separate HLMs were constructed for these two time points for each of the emotions. Thus, we used a total of 16 separate HLMs: one for each of eight possible emotions selected before and after the outcome. To meet the requirements of the model, we entered age, gender, and education as within-participant factors. We did not expect any differences to emerge as a function of these within-participant factors. HLM is a valuable tool for this analysis because it provides a powerful way to model data within nested structures; our data are composed of ratings nested within participants. Because of zero variance in some variables (e.g., reports of anger after a positive outcome), some higher order interaction terms needed to be omitted from analyses. Customized models for each emotion were constructed to include all mathematically estimable interactions.

For each of the emotions, we obtained results in the expected direction. That is, the probabilities of selecting each emotion were greatest in the scenario for which our model had predicted that emotion. Table 3 includes statistics and significance tests for all effects, and Tables 4 and 5 display the average proportion of emotions selected under each condition. The following sections discuss the major results for each of our theoretically predicted temporal categories.

Fear and hope: Prediction-related emotions. As noted earlier, some emotions are thought to be more prediction related, whereas others are thought to be more outcome dependent. The two emotions most associated with prediction are fear and hope. Whereas fear signals a potential danger that must be prepared for, hope signals a prediction that things may soon get better. As such, our model suggests that participants would use the fear and hope categories most at the time of prediction and less so after an outcome was known. Specifically, we predicted that the majority of variance in these categories would be accounted for through the main effect of prediction but only before outcome. Consistent with this hypothesis, we found that, compared with the no-prediction condition, people were most likely to select fear when predicting that something bad was about to happen, $t(39)=7.702, p<.001$; and less likely to select fear when predicting that something good was about to happen, $t(39)=-3.392, p=.001$. Consistent with the idea that, in some ways, hope is the inverse of fear, participants overwhelmingly chose hope when predicting that something good was about to happen, $t(39)=10.432, p<.001$. However, evidence for differentiation in the emotion categories was also present. For example, people also endorsed hope when predicting something bad, $t(39)=2.582, p=.01$. Furthermore, although a current bad situation was not by itself sufficient to indicate hope as the appropriate category, there was an interaction between current and prediction, indicating that people chose hope when the current situation was bad and the prediction was good, $t(39)=4.479, p<$ .001 , further implicating the importance of positive expectation in hope. Thus, unlike fear, which simply signals the prediction of something bad, hope (and its converse, hopelessness) may also

Table 4

Study 1 Means for Preoutcome Emotion Selections

\begin{tabular}{lccllllllll}
\hline Current & Prediction & Fear & Hope & Joy & Sad & Anger & Surprise & Content & Disgust & None \\
\hline Bad & Bad & $\mathbf{0 . 4 4 2}$ & 0.042 & 0.008 & 0.267 & 0.133 & 0 & 0.033 & 0.05 & 0.025 \\
Good & Bad & $\mathbf{0 . 5 0 8}$ & 0.075 & 0.042 & 0.117 & 0.075 & 0.033 & 0.075 & 0.033 \\
Bad & Good & 0.008 & $\mathbf{0 . 7 3 3}$ & 0.05 & 0.033 & 0.025 & 0.058 & 0.042 & 0.017 \\
Good & Good & 0.008 & $\mathbf{0 . 4 2 1}$ & $\mathbf{0 . 3 9 7}$ & 0 & 0.017 & 0.025 & 0.107 & 0.033 \\
Bad & None & 0.042 & 0 & 0.008 & $\mathbf{0 . 6 1 7}$ & 0.108 & 0.008 & 0.042 & 0.125 & 0.025 \\
Good & None & 0.033 & 0.025 & $\mathbf{0 . 3 7 5}$ & 0.025 & 0 & 0.017 & $\mathbf{0 . 4 5}$ & 0 \\
\hline
\end{tabular}

Note. Means that are significantly different from chance $(>0.31)$ are indicated in boldface type. 
Table 5

Study 1 Means for Postoutcome Emotion Selections

\begin{tabular}{|c|c|c|c|c|c|c|c|c|c|c|}
\hline Prediction & Outcome & Fear & Hope & Joy & $\mathrm{Sad}$ & Anger & Surprise & Content & Disgust & None \\
\hline $\mathrm{Bad}$ & As expected & 0.025 & 0.038 & 0.05 & 0.4 & 0.15 & 0 & 0.163 & 0.088 & 0.087 \\
\hline Good & As expected & 0.025 & 0.038 & 0.5 & 0.038 & 0 & 0.013 & 0.263 & 0.013 & 0.113 \\
\hline Nothing & As expected & 0 & 0.013 & 0.025 & 0.088 & 0 & 0.05 & 0.4 & 0.013 & 0.413 \\
\hline Bad & Better & 0.013 & 0.05 & 0.413 & 0.013 & 0.013 & 0.35 & 0.125 & 0.013 & 0.013 \\
\hline Good & Better & 0 & 0.013 & 0.55 & 0 & 0 & 0.4 & 0.038 & 0 & 0 \\
\hline Nothing & Better & 0 & 0.05 & 0.563 & 0 & 0.013 & 0.288 & 0.088 & 0 & 0 \\
\hline $\mathrm{Bad}$ & Worse & 0.138 & 0 & 0.013 & 0.5 & 0.188 & 0.063 & 0.025 & 0.063 & 0.013 \\
\hline Good & Worse & 0.038 & 0.025 & 0.026 & 0.329 & 0.342 & 0.102 & 0.051 & 0.076 & 0.013 \\
\hline Nothing & Worse & 0.138 & 0 & 0 & 0.4 & 0.288 & 0.063 & 0.038 & 0.05 & 0.025 \\
\hline
\end{tabular}

Note. Means that are significantly different from chance $(>0.31)$ are indicated in boldface type.

take into consideration how the future may contrast with the present.

Also consistent with theory, although fear and hope were still selected as outcome-based emotion categories, they were used significantly less often at outcome than at prediction: For fear, $t(39)=6.50, p<.001$; for hope, $t(39)=8.18, p<.001$. Specifically, people were more likely to select fear when the outcome was bad or worse than predicted, $t(39)=3.874, p<.001$; and marginally less likely to select fear when they had predicted something good, $t(39)=-1.650, p=.099$. Similarly, people were relatively less likely to choose hope when they had predicted something good and the outcome was better than expected, $t(39)=$ $-2.316, p<.05$. Because of the dynamic nature of emotion processing, with the processing of outcomes and predictions occurring simultaneously, and with each outcome implying a new prediction, it is likely that these results suggest a second-order prediction in the study. For example, after a good event, one does not need to be hopeful anymore, but after a bad outcome, one may remain in a state of fear by predicting additional bad outcomes. It should be noted, however, that these outcome-related effects were substantially smaller that the prediction-related effects, and neither fear nor hope was the most selected emotion for any of the outcome conditions.

Joy and sadness: Upward and downward affective trajectories. In addition to representing predictions, we have the ability to represent changes in our affective states. In other words, we can represent whether things are basically getting better or getting worse. These affective trajectories are important for understanding the state of the world and letting us know what types of preparation we need (or do not need) to make. Theoretically, we predict that joy and sadness reflect these changes. It is important to note that, although predictions may be part of a happy or sad episode, the critical element for labeling an emotion happy or sad simply requires a change in affective processing-one's affective state getting better or worse, respectively. Thus, in our study, we predict that joy and sadness will be most reported after the outcome, when participants learn that things have gotten better or worse. Consistent with this, we found that people selected joy more often when the current state was good, $t(39)=4.471, p<.001$; and when predicting something good, $t(39)=2.018, p<.05$; and less often when predicting something bad, $t(39)=-2.152, p<.05$. After the outcome, people used the joy category when they had predicted something good, $t(39)=4.694, p<.001$; and when the outcome was better than predicted, $t(39)=4.429, p<.001$; and they were unlikely to use it when the outcome was worse than expected, $t(39)=-4.068, p<.001$. Furthermore, we found that people endorsed sadness when the outcome was bad, $t(39)=4.869, p<$ .001 ; and that they were unlikely to endorse it when the outcome was good, $t(39)=-6.962, p<.001$. People also endorsed sadness when the current state was bad, $t(39)=7.413, p<.001$; and were unlikely to endorse it when predicting something good, $t(39)=$ $-7.131, p<.001$.

Anger and surprise: Dealing with the unexpected. What we expect to happen does not always happen, and it is critical to note the discrepancies between our internal and external worlds. For example, something bad can happen, but if it comes out of nowhere, it may signify something quite different than if it was expected. Conceptually, depictions of anger typically highlight discrepancies and violations. Thus, although anger was similar to sadness in that people were more likely to use the anger category more when the outcome was bad, $t(39)=5.111, p<.001$; and were less likely to use the anger category when the outcome was good, $t(39)=-2.743, p<.01$, we found that these effects interacted with the predictions that were provided. As demonstrated in Table 5, when contrasting sadness and anger, two emotion categories that were used often after a bad outcome, sadness followed an expected negative outcome whereas anger followed an unexpected negative outcome.

Surprise also involves a discrepancy between one's predictions and outcomes, although it does not necessarily carry the same negative connotation that anger does. Some have suggested that surprise may be a word that is used whenever something unexpected occurs (e.g., Feldman, 1995). We predicted that surprise would indeed be associated with an unpredicted change in trajectory; that is, surprise would be rated highly in situations in which the outcome was inconsistent with the prediction. Our predictions were confirmed to an extent; participants selected surprise both when the outcome was better than expected, $t(39)=5.535, p<$ .001 ; and when it was worse than expected, $t(39)=2.61, p<.01$. However, none of the interactions among time states were significant; we suspect that this may be due to the forced-choice response option that may have made selection of other, more commonly used emotion words (e.g., anger, joy) likely.

Contentment/calm: When nothing really changes. According to the affective trajectories hypothesis, a critical difference between moods and emotions reflect changes in emotional states. 
Thus, when there are no changes, no emotion or a sense of calm may follow. Following the model, we predicted that people would report contentment/calm in situations in which there was a positive current state, without necessarily experiencing any affective trajectory across time. In many ways, the use of the label calm is akin to a mild positive mood. Consistent with this prediction, we found that people were more likely to report contentment when the current state was good, $t(39)=2.212, p<.05$. Critically for the affective trajectories hypothesis, we found that people were less likely to report contentment when predicting either bad or good, $t(39)=-3.353, p=.001$; suggesting that they only reported contentment when predicting nothing, $t(39)=-2.781, p<.01$. Thus, effectively, people reported contentment most often when the current state was good and there was no prediction for the future. Consistent with these findings, we found that people were less likely to endorse contentment when the outcome was either better than expected, $t(39)=-4.305, p<.001$; or worse than expected, $t(39)=-3.368, p=.001$; or when, postoutcome, the current state and prediction had both been bad, $t(39)=-2.159$, $p<.05$.

However, one can also experience calm or contentment not only as a state of nothing of significance happening but also as the restoration of a positive state-a return to homeostasis-after an emotional experience. (Most people tend to feel mildly pleasant most of the time; Diener \& Diener, 1994.) This is a secondary aspect of calm: a sense of quiet and relaxation after a highly emotional episode. Under these conditions, the emotional experience subsides, and the positive mood can resume. Consistent with this idea, people were more likely to endorse contentment when they had predicted something bad and the outcome was better than expected, $t(39)=2.628, p<.01$.

Disgust. The affective trajectories hypothesis predicts no systematic effects for disgust (see Table 1), and it was included for complete representation of common emotion terms. In this study, we found that, before the outcome, people were more likely to endorse disgust if the current state was bad, $t(716)=4.842$; and less likely if predicting good, $t(716)=-3.897$, both $p \mathrm{~s}<.001$. After the outcome, people were more likely to select disgust if they had predicted something bad, $t(708)=2.208, p<.05$; and less likely if the outcome was good, $t(708)=-2.701, p<.01$. There was also a marginally significant interaction, indicating that people were somewhat less likely to select disgust if they had predicted bad and the outcome was worse than expected. However, as indicated in Tables 4 and 5, no selection of disgust was endorsed at a rate greater than chance $($ chance $=0.11$; significance $=0.31$ ). Disgust operates differently from other emotions, perhaps because it is tied specifically to the stimulus that elicits it whereas other emotions are tied to the way we relate with our environments.

\section{Discussion}

This is the first demonstration that emotion categories can be reliably reported in the absence of any semantically detailed cognitive appraisal. Mere information regarding one's affective trajectory was sufficient to indicate the most appropriate emotion category. For example, nearly every time participants read a situation in which a negative prediction was made, participants indicated fear as the emotion category that best applied. Participants rarely indicated sadness or anger at this time, using these catego- ries primarily when a worse-than-expected outcome occurred. This indicates a high degree of discrimination matching specific situational cues to specific emotion labels. This suggests that emotion categories may serve as cues for simpler comparison processes that function as core affective ingredients. Thus, emotion words are not arbitrary but are systematically organized to reflect the fundamental processes that construct them-the dynamic interaction of multiple valence systems. Whereas previous theories have conceptualized emotions alternately as static modules, cognitive constructions, or dual-process patterns of activation, this study provides a robust demonstration of the way affective trajectories and their interactions underlie the emotion labels used in everyday life. That is, our emotion words may function as placeholders for describing the rich affective topography that underlies them-including our affective trajectories through time.

\section{Study 2: Ratings}

Study 1 is the first to demonstrate that, given decontextualized information about affective movement through time, participants could reliably and systematically select emotion labels for each scenario in a way that is consistent with theory. Psychological constructivism (e.g., Barrett, 2009) suggests that this is how we decode the emotions we experience: Given a particular affective state, we impose an emotion label that then allows that state to be interpreted as consistent with that label. However, when one does not choose to interpret one's affective state as one specific emotion, affect can be experienced in multiple ways. For example, a general negative state can contain elements of sadness, anger, and fear, but that affective state is experienced as one emotion only by imposing the label sad, angry, or afraid. Thus, if emotions are labels used to categorize ongoing affective experience, we may use multiple labels to capture the unique elements that give rise to affective experience.

The way we interpret an experience may depend on the weight we give to specific aspects of our affective trajectories. This implies that different experiences may contain common elements because of activation in different subsystems; for example, two different experiences of sadness and anger can both contain traces of fear, as long as a negative prediction is being made while also processing a negative current situation. There are many blends possible, and people sometimes do report feeling mixed emotions. Rather than indicating two separate feeling states, this may rather reflect the ability to label an affective state in multiple ways. Participants in Study 1 systematically differentiated the emotions they would feel in each situation when asked to choose one label, but given the complex nature of affective experience, participants asked to provide ratings of all the emotion labels should give more variable responses that allow for the possibility of mixed emotional experiences, such as designating a situation as containing elements of both sadness and fear or both joy and contentment. Although this suggests a more complex categorization process, our hypothesis remains the same. Specifically, we predict that differences in the emotions reported will reflect the types of computations that are involved in the processing of ongoing affect.

To examine these questions, we conducted a second study in which participants used a rating scale to indicate the extent to which each emotion was represented in the scenario. We expected participants' ratings to be less uniform under these conditions, in 
accordance with the predictions of psychological constructivist models suggesting that emotions are only strongly differentiated when a categorization scheme is imposed on an affective state. Furthermore, this approach allows us to statistically control for the influence of the circumplex dimensions to examine whether additional variance in emotions can be explained with our variables. If emotions are best explained by the dimensional model, then affective trajectories should contribute no additional information toward explaining emotion ratings beyond the dimensions revealed through factor analysis. However, if our model is a useful way to represent emotion, it should contribute toward explaining emotion ratings beyond the influence of the circumplex dimensions.

\section{Method}

Participants. Thirty-one undergraduate students (13 female, 18 male; age range $=18-23$, mean age $=18.6$ ) participated in exchange for course credit in an introductory psychology class.

Procedure. The procedure was identical to the procedure for Study 1, except for the following change: Rather than selecting just one emotion that best fit the scenario, participants rated the extent to which each of the emotion words fit how they would be feeling in that scenario using a rating scale ranging from 1 (not at all) to 7 (perfectly). The emotion words were the same as in Study 1, except that "no emotional response" was no longer an option (as participants could simply give all emotions a rating of 1 if they felt that none were applicable). As with Study 1, ratings were made before and after the outcome for each scenario. There were 18 trials, and participants made 288 ratings.

\section{Results}

Previous research has suggested that ratings of discrete emotions can be rerepresented as coordinates within a two-dimensional space. That is, rather than considering a participant's rating of anger, fear, or sadness as reflecting distinct representations, each rating reflects the difference between a person's core affective state and the conceptual location of a prototypical emotion within a circumplex structure. For example, if a person is feeling negative with a high degree of arousal, she might rate her fear as high (a high arousal, negative emotion), her sadness as moderate (a low arousal, negative emotion), and her calmness as low (a low arousal, positive emotion). As this example shows, each emotion rating reflects a comparison between a real affective state (high arousal and negative) with a conceptual two-dimensional mapping.

Replicating this factor structure, a principal-components factor analysis of the emotion rating data found two factors with eigenvalues greater than 1 that were able to account for $68 \%$ of the variance. Allowing for a third factor (eigenvalue $=0.68$ ) accounted for only an additional $0.08 \%$ and did not add to the interpretability of the factor structure. In accordance with previous work (Russell, 1980), we used the canonical two-factor solution for all further analyses. After a varimax rotation of the two extracted factors, we labeled the resulting factor structure positive affect (PA) and negative affect (NA). The PA factor was anchored by 0.87 on the high end and -0.55 on the low end. The NA factor was anchored by 0.78 on the high end and -0.26 on the low end.

Although the two-dimensional model was able to account for $68 \%$ of the variance in participants' ratings of emotion categories, our model predicts that additional variance can be accounted for by understanding the affective trajectories inherent in our affective topography. Specifically, emotion categories are used not only to represent core affect but also the temporal changes that lead to subjective emotional experiences. To examine this question, we constructed a series of mixed models in SAS predicting the selected emotion categories. Continuous emotion ratings were predicted by our manipulated variables: current state, prediction, and outcome. All interaction terms were modeled in addition to the main effects. Because we had separate predictions for the emotions that would be labeled at the time of prediction and those after the outcome, separate mixed models were constructed for these two time points for each of the emotions. To more conclusively demonstrate that affective trajectories are a uniquely predictive part of the emotion prototype, we also entered the factor scores of PA and NA (and the interaction) into the models. Table 6 includes statistics and significance tests for all effects, and Tables 7 and 8 display the average rating of each emotion for each condition. The following sections discuss the major results for each of our theoretically predicted temporal categories.

Fear and hope: Prediction-related emotions. As demonstrated in Study 1, participants were likely to endorse high levels

Table 6

Study 2 Effects for Emotion Ratings Made Pre- and Postoutcome

\begin{tabular}{|c|c|c|c|c|c|c|c|c|}
\hline Effect & Fear & Hope & Joy & Sadness & Anger & Surprise & Content & Disgust \\
\hline \multicolumn{9}{|l|}{ Preoutcome } \\
\hline Current status & $7.44^{*}$ & $6.40^{*}$ & $12.29^{* * * *}$ & $6.21^{*}$ & 1.94 & 0.01 & 1.07 & $3.00^{\dagger}$ \\
\hline Prediction & $11.42^{* * * *}$ & $8.03^{* * * *}$ & $5.83^{* *}$ & $5.34^{* *}$ & $2.87^{\dagger}$ & 1.11 & $9.36^{* * * *}$ & 1.72 \\
\hline Current Status $\times$ Prediction & 2.51 & $2.86^{\dagger}$ & $6.34^{* *}$ & $3.01^{\dagger}$ & 0.38 & 0.75 & 0.48 & 1.73 \\
\hline \multicolumn{9}{|l|}{ Postoutcome } \\
\hline Current status & 0.53 & 0.05 & 0.00 & $6.13^{*}$ & 0.47 & 0.16 & 0.49 & 0.07 \\
\hline Prediction & 0.24 & 1.58 & 0.70 & 2.74 & 0.16 & $4.03^{*}$ & $2.48^{\dagger}$ & 0.22 \\
\hline Current Status $\times$ Prediction & 1.98 & 1.09 & 0.14 & 0.15 & 0.32 & 0.24 & 0.37 & $2.70^{\dagger}$ \\
\hline Outcome & $4.31^{*}$ & $6.06^{* * *}$ & $11.57^{* * * *}$ & $11.77^{* * *}$ & 1.53 & $33.61^{* * *}$ & $19.79^{* * * *}$ & $3.80^{*}$ \\
\hline Current Status $\times$ Outcome & 1.33 & 0.53 & 1.89 & 1.08 & 1.05 & 2.66 & 0.02 & 1.24 \\
\hline Prediction $\times$ Outcome & $2.27^{\dagger}$ & $2.21^{\dagger}$ & $4.15^{* *}$ & 0.76 & $3.94^{* * *}$ & 1.34 & 2.38 & $2.85^{*}$ \\
\hline Current Status $\times$ Prediction $\times$ Outcome & 1.64 & 0.09 & 1.17 & 1.44 & 0.81 & 0.85 & 0.45 & 0.46 \\
\hline
\end{tabular}

Note. Study 2 effects are reported as $F$ values.

${ }^{\dagger} p<.10 .{ }^{*} p<.05 .{ }^{* *} p<.01 .{ }^{* * *} p<.001$. 
Table 7

Study 2 Means for Emotion Ratings Made Preoutcome

\begin{tabular}{|c|c|c|c|c|c|c|c|c|c|}
\hline Current & Prediction & Fear & Hope & Joy & Sad & Anger & Surprise & Content & Disgust \\
\hline Bad & Bad & 4.83 & 3.08 & 1.56 & 4.97 & 4.78 & 3.35 & 1.99 & 3.43 \\
\hline Good & Bad & 3.94 & 4.09 & 3.33 & 3.06 & 2.95 & 3.37 & 3.30 & 2.37 \\
\hline Bad & Good & 3.11 & 5.13 & 3.66 & 2.86 & 2.96 & 3.20 & 3.22 & 2.04 \\
\hline Good & Good & 2.04 & 5.16 & 5.58 & 1.68 & 1.51 & 3.30 & 3.91 & 1.52 \\
\hline $\mathrm{Bad}$ & None & 4.06 & 3.02 & 1.67 & 5.33 & 4.86 & 3.45 & 2.29 & 3.40 \\
\hline Good & None & 1.65 & 4.74 & 5.89 & 1.46 & 1.47 & 3.38 & 4.71 & 1.40 \\
\hline
\end{tabular}

Note. Values in boldface type represent predicted effects; italicized values represent secondary effects.

of fear when predicting a negative future, $F(2,60)=11.42, p<$ .001. Thus, fear is highly dependent on the prediction of negative future events. Similarly, as also demonstrated in Study 1, we found that people felt most hopeful when predicting positive things for the future, $F(2,60)=8.03, p<.001$. There was also a marginally significant interaction between current state and prediction, so that people rated hope more highly when the current state was bad and people were making a positive prediction for the future, $F(2,60)=$ $2.86, p=.065$. Thus, as in Study 1 , hope and fear may not be reciprocals of one another, with hope (and hopelessness) potentially using a contrast across time points; that is, imagining how much brighter the future could be in comparison with the negative present.

Additionally, unlike our results from Study 1, people rated fear highly when the outcome was worse than expected, $F(2,60)=$ $4.31, p<.05$; and hope when the outcome was better than expected, $F(2,60)=6.06, p<.01$. As the present study allows for greater fluidity of interpretation, these ratings indicate that participants may have made the natural inference that bad things that happen now may indicate more bad things to come (fear) or that good things that happen now may indicate more good things to come (hope). Thus, a new prediction based on the current outcome may be implicit in their ratings.

Joy and sadness: Upward and downward affective trajectories. We found that people rated joy as being highly representative of the emotion they would feel when the outcome was better than predicted, $F(2,60)=11.57, p<.001$; particularly when a better-than-predicted outcome followed a negative prediction, $F(4$, $120)=4.15, p<.01$. The results from before the outcome also supported these findings; people anticipated feeling greater joy when the current state was good, $F(1,30)=12.29$; and they predicted that good things would happen in the future, $F(2,60)=$ 5.83; resulting in a significant interaction; $F(2,60)=6.34$, all $p s<.01$. The preoutcome results may also indicate a trajectory extending back in time: Ratings of joy were high when the current state was good, and a positive current state is typically a result of a previously positive outcome, which may have been informed by previous predictions, and so forth. In summary, people rated joy more highly when there was a positive affective trajectory over time.

The results for sadness were also consistent with those in Study 1. Participants rated sadness more highly for outcomes that were worse than predicted, $F(2,60)=11.77, p<.001$. After the outcome, people also indicated higher ratings of sadness when the previous current state had been bad, $F(1,30)=6.13, p<.05$; and they had predicted a negative future, $F(2,60)=2.74, p=.073$. These results suggest that sadness is associated with a consistent negative pattern of events across time. Before the outcome, people gave higher ratings of sadness when the current state was bad, $F(1$, $30)=6.21, p=.05$; and they had no predictions for the future, $F(2,60)=5.34, p<.01$; these findings were supported by a marginally significant interaction, $F(2,60)=3.01, p=.057$. Thus, before the next emotionally significant event, sadness is often a function of the current state (which is arguably a function of the outcome that directly preceded it) and is focused on the events that produced that state.

Additionally, unlike our results for Study 1, we found that people rated sadness highly when predicting something bad would happen in the future, $F(2,60)=5.43, p<.01$. This is consistent with our idea that sadness is represented as a negative or downward trajectory: Expecting things to be worse in the future than they are in the present indicates a worsening of affective state over

Table 8

Study 2 Means for Emotion Ratings Made Postoutcome

\begin{tabular}{|c|c|c|c|c|c|c|c|c|c|}
\hline Prediction & Outcome & Fear & Hope & Joy & Sad & Anger & Surprise & Content & Disgust \\
\hline Bad & As expected & 3.47 & 3.13 & 2.45 & 4.45 & 4.40 & 2.87 & 2.84 & 3.05 \\
\hline Good & As expected & 1.69 & 4.18 & 5.47 & 1.61 & 1.48 & 3.45 & 4.61 & 1.52 \\
\hline Nothing & As expected & 2.27 & 3.08 & 3.48 & 2.77 & 2.71 & 2.92 & 4.08 & 2.19 \\
\hline Bad & Better & 1.81 & 4.48 & 6.31 & 1.44 & 1.45 & 5.16 & 4.34 & 1.35 \\
\hline Good & Better & 1.90 & 4.32 & 5.90 & 1.50 & 1.60 & 5.11 & 4.21 & 1.55 \\
\hline Nothing & Better & 2.00 & 4.52 & 6.24 & 1.60 & 1.81 & 5.50 & 4.55 & 1.60 \\
\hline Bad & Worse & 4.44 & 2.40 & 1.71 & 5.81 & 5.27 & 4.53 & 1.97 & 4.31 \\
\hline Good & Worse & 3.87 & 2.45 & 1.44 & 5.55 & 5.65 & 5.03 & 1.81 & 3.55 \\
\hline Nothing & Worse & 4.45 & 2.47 & 1.48 & 5.71 & 5.23 & 4.58 & 1.74 & 3.68 \\
\hline
\end{tabular}

Note. Values in boldface type represent predicted effects; italicized values represent secondary effects. 
time. It is also consistent with the differences between Study 1 and Study 2 for fear and hope: Implicit in participants' ratings may be the assumption that when a particular affective trajectory has begun it will continue in the same direction (e.g., things going poorly will continue to do so). However, it should be noted that the average rating for fear was higher than the average rating for sadness when predicting something bad, $M \mathrm{~s}=4.39$ (fear) and 4.02 (sadness), $F(1,60)=10.20, p<.01$, indicating that fear was still the dominant response when predicting something bad.

Anger and surprise: Dealing with the unexpected. Participants rated anger more highly when the outcome was worse than predicted, $F(4,120)=3.94, p<.01$. Differentiating from sadness, which was also indicated when the outcome was worse than predicted, anger was rated lower when a negative outcome had been predicted $(M=5.27)$ than when a positive outcome had been predicted $(M=5.65)$, whereas sadness was rated higher when a negative outcome had been predicted $(M=5.81)$ than when a positive outcome had been predicted $(M=5.55), F(1,92)=5.59$, $p<.05$. Thus, anger seems to hinge on a violation of expectation: When predicting bad things that then came to pass, people rated anger relatively low, whereas if they predicted good things but instead events took a turn for the worse, people rated anger more highly. In addition, we found that people rated surprise highly only after the outcome but, unlike in Study 2, after both positive and negative unexpected outcomes, $F(2,60)=33.61, p<.001, M \mathrm{~s}=$ 5.26 (better than expected) and 4.71 (worse than expected). This suggests that the results of Study 1 may be partially an consequence of the forced-choice format. People can feel surprised in response to both positive and negative unexpected outcomes. When asked to select a single emotion word, they are more likely to select anger in response to a negative unexpected outcome, but when given the option to respond more freely, participants can report that they might feel surprised and joyful or surprised and angry. Current state also mattered: Holding prediction constant, participants rated surprise highly when the current state was incongruous with the outcome (i.e., current bad/outcome good or current good/outcome bad), $F(2,60)=2.66, p=.078 ; M \mathrm{~s}=4.87$ and 4.80 , respectively. Thus, surprise was rated highly for situations in which an unpredicted change in trajectory occurred. Surprise seems to be a general term that can be used to describe any unexpected outcome in conjunction with the emotion resulting from that outcome.

Contentment/calm: When nothing really changes. People reported relatively high levels of contentment when the outcome was neutral or as predicted, $F(2,60)=19.79, p<.001$. In addition, people reported that they would experience a greater sense of contentment and/or calm when making no particular prediction for the future, $F(2,60)=9.36, p<.001$. A marginally significant interaction indicated that people rated contentment more highly when they had made no prediction and the outcome was an ordinary/neutral event, $F(4,120)=2.38, p=.056$. This particular interaction reflects no change across time. Thus, people endorsed contentment/calm for situations in which nothing emotionally significant was occurring. These data provide further evidence that contentment is a present-focused affective state.

Disgust. The affective trajectories hypothesis predicts no systematic effects for disgust (see Table 2), and we found that ratings of disgust did not lend themselves to any theoretically interpretable pattern of data. For example, people endorsed higher levels of disgust when the outcome was neutral or as predicted, $F(2,60)=3.80, p<.05$; whereas people also rated disgust more highly for scenarios in which they had predicted something good and that prediction then came true, $F(4,120)=2.85, p<.05$. Thus, significant effects were scattered, inconsistent, had lower effect sizes than those for ratings of any other emotion, and did not easily lend themselves to any logical interpretation.

\section{Discussion}

For the most part, when participants had the opportunity to assign multiple emotion categories (to different degrees) to each of the scenarios, ratings of appropriateness were highest where our model predicted them. For example, in response to a negative prediction, participants rated fear as an appropriate emotion, whereas after a better-than-expected outcome, participants rated joy as appropriate. However, the degree of differentiation among the emotions within any particular scenario was not as pronounced as we found in Study 1. For example, although participants indicated that, when currently feeling negative, fear would be an expected response to a negative prediction, participants also rated sadness and anger as appropriate to the same degree. Similarly, not only were sadness and anger rated as appropriate after a worse than expected outcome, but so was fear.

One theoretical explanation for this apparent discrepancy is that when participants were not compelled to categorize the affective state into a single discrete emotion, they may have been open to the multiple emotional states that could be appropriate. Additional aspects of the situation may be considered, and participants may have been sensitive to the various states that are possible. For example, when allowed to consider which emotions might be possible, participants may have considered additional time points in affective space. That is, although this information was not provided to the participants, they may have considered the events that led to a particular combination or what would be logically expected to follow. Take, for example, when participants consider a worse-than-expected outcome. Participants have the option of considering the current aspects of the emotion (the affective now) or the possible consequences of what this might mean (the affective future). That is, a negative outcome may be understood in the present, leading to sadness or anger, or it may be a sign of additional negative effects, leading to predictions of additional negative consequences and, therefore, fear. Such a combination may reflect a "mixed" emotional state-sadness or anger in the present but fear for the future. Similarly, consider the prediction of something bad. Unlike in Study 1, in which all participants reported fear as the single appropriate emotion, sadness and anger were also reported but only when the current state was bad. There is a common thread of fear across these conditions, but the type of fear as a mixed state may differ dramatically. Thus, although these are all "fear," these fear states may be qualitatively and quantitatively distinct. Fear mixed with sadness is different from fear mixed with anger (Gray, 1982).

Inherent in the idea of affective trajectories is the suggestion that affective states preceding the current state (which contained predictions of their own) are critical for an understanding of the emotional state of the person in the current situation. Thus, just as different temporal foci can cause a negative outcome to result in a present-focused emotion ("I feel bad") or a past-focused emotion 
("this is not what I expected"), a negative current state can also be present focused ("I feel bad"), past focused ("what got me here"), or future focused ("given this, what I think will happen next"). Study 2 may have allowed participants to demonstrate these more nuanced emotional states. Indeed, there may even be the possibility of shifts between emotion categories by shifting attention from one affective system to another. By focusing more on the negative aspects of the predicted state than the positive aspects of the current state, this may lead to an emotion such as fear (e.g., "regardless of how good I feel now, things will soon be bad"), whereas if the reverse is true-if we focus on the positive current state more than on our negative prediction-this may lead to an emotion such as hope (e.g., hoping things continue to go well despite the odds). Future research should directly manipulate these differences in temporal focus.

The aforementioned suggestions notwithstanding, it should be acknowledged that valence does play a major role in the rating of emotions: When given a trajectory that would cause a negative emotion, all negative emotions are endorsed to some extent, whereas the same is true for positive emotions. Indeed, evaluating objects or situations as pleasant or unpleasant may be a fundamental way of categorizing human experience. However, even within this general effect of valence, there still is evidence of differentiation among emotion categories on the basis of trajectory information.

\section{General Discussion}

Emotion categories are a useful way for people to organize and understand their ongoing subjective experience, as well as explain that subjective state to others. Through associating specific categories with ongoing affective experience, we develop a richer understanding of our place in the world. However, where do these categories come from and what do they represent? Explanations have ranged from rich cognitive schemes to biological modules, to representations of simple valence and arousal. In these studies, we demonstrated that information about affective movement through time is represented within emotion categories. Thus, the varied language of emotion may be indicative of more simple changes in our ongoing state. This is a crucial difference from previous theory. Our studies show that people can differentiate among major emotion categories on the basis of information about affective trajectories alone, and a model of emotion including these trajectories accounts for more variability in emotion ratings than the current dominant model alone. These data suggest that emotion categories, at least in part, may represent a distributed pattern of affective information. The potential patterns reflect an affective topography, with unique patterns labeled as emotions (if such a labeling opportunity arises). This conceptualization highlights the dynamic nature of emotion and how emotions may transform from one state (with a specific label) to another. Thus, shifts in emotion from, say, fear to sadness to anger, are easily accomplishedsomething that would be far more difficult if a modular basic emotion architecture existed.

It is critical that further study of emotion accurately accounts for the way that we absorb and process information in our environments. The iterative reprocessing model (Cunningham \& Zelazo, 2007) represents the way researchers evaluate information on a microscopic time scale, continually updating and reprocessing that information in successive iterations as we improve in our ability to comprehend it. To fully explain the psychological construction of subjective emotional states, the affective trajectories hypothesis extends this idea by considering how these dynamically shifting cognitive processes affect our emotional representations. The present work provides evidence that people can appreciate the way in which these processes interact to shape specific emotional experiences, thus providing support for a new model of emotion that both clarifies and integrates previous models. For example, we found that fear and hope were both dependent on predictions for the future: Fear is associated with predicting something bad, whereas hope is associated with predicting something good. Joy and contentment, although both positive feeling states, differed in that joy was associated with improvement over time whereas contentment/calm was associated with no change over time. Additionally, although both anger and sadness were related to a negative outcome, anger was chosen or rated less often relative to sadness in situations in which that negative outcome had been predicted. Anger and surprise were both related to unexpected changes in trajectory over time; surprise was more likely than anger to be associated with a positive change (Study 1), but surprise was also readily applied to situations with a negative change (Study 2). These findings are consistent with the location of surprise on the affective circumplex: high in arousal but effectively neutral in valence (e.g., Russell, 2003), as it can be used to describe either positive or negative events.

Although our studies focus on the emotion labels that people would use to describe situations, we believe that these labels are used because they map onto emotional experience. The reason that people label a situation as being fearful is that they would label their emotional experience similarly if in that situation. However, it is also possible that these labels represent the conceptual information that people have developed about emotion and reflect prototypical knowledge rather than separable building blocks of emotional experience.

Future work with neuroscience methods such as functional MRI will be necessary for distinguishing between these competing hypotheses (Kirkland \& Cunningham, in press). Furthermore, methods such as experience sampling will be useful for determining whether emotional experience follows from the same principles.

Emotions are linked to a particular set of stimulus events, whether generated internally or experienced externally. New information (or changes in information) is processed with regard to its affective meaning and its relevance for affective trajectory, functioning as a type of "preappraisal" in priming the system toward a particular interpretation. After this initial processing, people may activate particular cognitive appraisals and behavioral tendencies to help define the situation and its relevance for the self. These higher order processes function to further differentiate and delineate our feeling state, leading to categorization and labeling. By activating the appropriate category, more rich semantic and episodic information becomes available to allow for flexible solutions to potential challenges and opportunities. As time goes on, this process repeats, allowing for additional processing, composed of more conscious appraisals, correction, and refinement. Thus, despite the flexibility of the system, an emotion, once labeled, may take on a life of its own. That is, once an individual has labeled her affective state as anger, information will be processed to be con- 
gruent with this impression, and an "anger" emotion will remain despite shifts in affective processing. Moreover, through top-down processes, the affective state that led to the assessment of anger may be foregrounded, leading to a more continuous and maintained emotional state. On this view, although we have highlighted the bottom-up, situationally defined generation of emotion, through top-down processes, we can bias, maintain, or reinterpret our situations to lead to different patterns in affective space. Indeed, cognitive reappraisal (Goldin, McRae, Ramel, \& Gross, 2008) requires people to change their interpretation of an outcome to be more or less positive. We cannot only change our understanding of the outcomes, but we can "misremember" our previous affective state or even what we predicted to happen as forms of emotional regulation. These changes in affective representation should lead to different comparisons, and, if we are open to it, a new emotional label.

This framework can be used to reinterpret patterns of neural activity that have been previously interpreted as reflecting basic emotion circuits. For example, one of the most replicated results in affective neuroscience is the fear-amygdala relationship (Whalen et al., 1998). However, given the amygdala's role in not only fear but also other processes related to learning evaluative associations and generating automatic responses to specific cues (LaBar, Gatenby, Gore, LeDoux, \& Phelps, 1998), we suggest that the amygdala represents not fear per se but rather a prediction signaling an anticipated negative event (see Cunningham, Haas, \& Jahn, in press). Thus, the fear-amygdala link can be reinterpreted in terms of affective trajectory: Fear is the result of the prediction that something bad will occur. Similarly, subjective reports of sadness and happiness tend to be associated with differences in orbitofrontal cortex processing (Mayberg et al., 1999; Kringelbach \& Berridge, 2009) - areas that have also been found to be involved in the representation of experiencing outcomes (Cunningham, Kesek, \& Mowrer, 2009; Wunderlich, Rangel, \& O’Doherty, 2009). This is consistent with the idea that happiness and sadness reflect changes in one's current state as a function of one's outcomes. Taking this idea one step further, anger is associated with activity in both the orbitofrontal cortex and the network comprising the amygdala and basal ganglia (Davidson, Putnam, \& Larson, 2000; Dougherty et al., 1999). It is not that the orbitofrontal cortex, amydala, and basal ganglia are all "anger areas," but rather we suggest that these links exist because anger requires information about both the prediction and one's outcomes after that prediction.

Because emotions may result from the categorization of the interactions between several affective systems, this suggests that there may be a near-infinite number of affective states that can potentially be labeled as different emotions. This conceptualization is similar to a proposal by Plutchik (1980), who suggested that many emotions are combinations of more fundamental elements in a manner analogous to a color wheel. Just as we label (and see) continuous visual input with discrete color names such as orange, teal, and violet, so too the affective input that we receive is continuous, fluid, and subject to blending and multiple potential interpretations. Some affective states may be classified as one emotion or another arbitrarily, an individual's emotion vocabulary can dictate the range of responses, and even personality variables may determine where the cutoffs are. In this manner, language interacts with affect to construct our emotions. The categories that we use to describe emotions are not the result of a specialized emotion system, nor are they arbitrary categories reflecting only linguistic conventions while being removed from more basic psychological processes. Instead, influenced by our affective trajectories, we flexibly categorize and transform our affective states into emotions. Once categorized, those emotions may continue to develop and unfold in a way that is consistent with that categorization. In this manner, the rich tapestry of emotional experience may begin from processes as simple as our subjective trajectories through time.

\section{References}

Adolphs, R., Russell, J. A., \& Tranel, D. (1999). A role for the human amygdala in recognizing emotional arousal from unpleasant stimuli. Psychological Science, 10, 167-171.

Adolphs, R., Tranel, D., Damasio, H., \& Damasio, A. (1994). Impaired recognition of emotion in facial expressions following bilateral damage to the human amygdala. Nature, 372, 669-672.

Arnold, M. B. (1960). Emotion and personality. New York: Columbia University Press.

Barrett, L. F. (2006). Are emotions natural kinds? Perspectives on Psychological Science, 1, 28-58.

Barrett, L. F. (2009). The future of psychology: Connecting mind to brain. Perspectives on Psychological Science, 4, 326-339.

Barrett, L. F., Mesquita, B., \& Gendron, M. (in press). Emotion perception in context. Current Directions in Psychological Science.

Bechara, A., Tranel, D., Damasio, H., Adolphs, R., Rockland, C., \& Damasio, A. R. (1995). Double dissociation of conditioning and declarative knowledge relative to the amygdala and hippocampus in humans. Science, 269, 1115-1118.

Biederman, I. (1987). Recognition-by-components: A theory of human image understanding. Psychological Review, 94, 115-147.

Cacioppo, J. T., \& Tassinary, L. G. (1990). Inferring psychological significance from physiological signals. American Psychologist, 45, 16-28.

Calder, A. J., Keane, J., Manes, F., Antoun, N., \& Young, A. W. (2000). Impaired recognition and experience of disgust following brain injury. Nature Neuroscience, 3, 1077-1078.

Carroll, J. M., \& Russell, J. A. (1996). Do facial expressions signal specific emotions? Judging emotion from the face in context. Journal of Personality and Social Psychology, 70, 205-218.

Carver, C. S. (1979). A cybernetic model of self-attention processes. Journal of Personality and Social Psychology, 37, 1251-1281.

Carver, C. S., \& Scheier, M. E. (1990). Origins and functions of positive and negative affect: A control-process view. Psychological Review, 97, 19-35.

Clore, G. L., \& Huntsinger, J. R. (2009). How the object of affect guides its impact. Emotion Review, 1, 39-54.

Clore, G. L., Ortony, A., \& Foss, M. A. (1987). The psychological foundations of the affective lexicon. Journal of Personality and Social Psychology, 53, 751-766.

Cunningham, W. A., Haas, I. J., \& Jahn, A. (in press). Attitudes. In J. Decety \& J. T. Cacioppo (Eds.), Handbook of social neuroscience. New York: Oxford University Press.

Cunningham, W. A., \& Van Bavel, J. J. (2009). Varieties of emotional experience: Difference in object or computation. Emotion Review, 1, $56-57$.

Cunningham, W. A., Van Bavel, J. J., \& Johnsen, I. R. (2008). Affective flexibility: Evaluative processing goals shape amygdala activity. Psychological Science, 19, 152-160.

Cunningham, W. A., \& Zelazo, P. D. (2007). Attitudes and evaluations: A social cognitive neuroscience perspective. Trends in Cognitive Sciences, 11, 97-104.

Cunningham, W. A., \& Zelazo, P. D. (2009). The development of iterative reprocessing: Implications for affect and its regulation. In P. D. Zelazo, 
M. Chandler, \& E. Crone (Ed.), Developmental social cognitive neuroscience (pp. 81-98). New York: Psychology Press.

Cunningham, W. A., Kesek, A., \& Mowrer, S. (2009). Distinct orbitofrontal regions encode stimulus and choice valuation. Journal of Cognitive Neuroscience, 21, 1956-1966.

Davidson, R. J., Putnam, K. M., \& Larson, C. L. (2000). Dysfunction in the neural circuitry of emotion regulation: A possible prelude to violence. Science, 289, 591-594.

Diener, E., \& Diener, C. (1994). Most people are happy. Psychological Science, 7, 181-185.

Dougherty, D. D., Shin, L. M., Alpert, N. M., Pitman, R. K., Orr, S. P., Lasko, M., ... Rauch, S. L. (1999). Anger in healthy men: A PET study using script-driven imagery. Biological Psychiatry, 46, 466-472.

Ekman, P., \& Friesen, W. V. (1971). Constants across cultures in the face and emotion. Journal of Personality and Social Psychology, 17, 124129.

Elliott, R., \& Dolan, R. J. (2003). Functional neuroimaging of depression: A role for medial prefrontal cortex. In R. J. Davidson, K. R. Scherer, \& H. H. Goldsmith (Eds.), Handbook of affective sciences (pp. 117-125). New York: Oxford University Press.

Feldman, L. (1995). Variations in the circumplex structure of mood. Personality and Social Psychology Bulletin, 21, 806-817.

Garavan, H., Pendergrass, J. C., Ross, T. J., Stein, E. A., \& Risinger, R. C. (2001). Amygdala response to both positively and negatively valenced stimuli. NeuroReport, 12, 2779-2783.

Gendron, M., \& Barrett, L. F. (2009). Reconstructing the past: A century of ideas about emotion in psychology. Emotion Review, 1, 316-339.

Goldin, P. R., McRae, K., Ramel, W., \& Gross, J. J. (2008). The neural bases of emotion regulation: Reappraisal and suppression of negative emotion. Biological Psychiatry, 63, 577-586.

Gray, J. A. (1982). The neuropsychology of anxiety: An enquiry into the functions of the septo-hippocampal system. New York: Oxford University Press.

Hamann, S. B., Ely, T. D., Hoffman, J. M., \& Kilts, C. D. (2002). Ecstasy and agony: Activation of the human amygdala in positive and negative emotion. Psychological Science, 13, 135-141.

Jarvis, B. G. (2006). MediaLab (Version 2006.2.19). New York: Empirisoft.

Johnson-Laird, P. N., \& Oatley, K. (1989). The language of emotions: An analysis of a semantic field. Cognition \& Emotion, 3, 81-123.

Kirkland, T., \& Cunningham, W. A. (in press). Neural basis of affect and emotion. Wiley Interdisciplinary Reviews: Cognitive Sciences.

Kringelbach, M. L., \& Berridge, K. C. (2009). Towards a functional neuroanatomy of pleasure and happiness. Trends in Cognitive Sciences, 13, 479-487.

Kringelbach, M. L., \& Rolls, E. T. (2004). The functional neuroanatomy of the human orbitofrontal cortex: Evidence from neuroimaging and neuropsychology. Progress in Neurobiology, 72, 341-372.

LaBar, K. S., Gatenby, J. C., Gore, J. C., LeDoux, J. E., \& Phelps, E. A. (1998). Human amygdala activation during conditioned fear acquisition and extinction: A mixed-trial fMRI study. Neuron, 20, 937-945.

Lazarus, R. S. (1982). Thoughts on the relations between emotion and cognition. American Psychologist, 37, 1019-1024.

Lazarus, R. S. (1984). On the primacy of cognition. American Psychologist, 39, 124-129.

Lindquist, K., \& Barrett, L. F. (2008). Constructing emotion: The experience of fear as a conceptual act. Psychological Science, 19, 898-903.

Lindquist, K., Barrett, L. F., Bliss-Moreau, E., \& Russell, J. A. (2006). Language and the perception of emotion. Emotion, 6, 125-138.

Lindquist, K. A., Wager, T. D., Kober, H., Bliss-Moreau, E., \& Barrett, L. F. (in press). The brain basis of emotion: A meta-analytic review. Behavioral and Brain Sciences.

Mayberg, H. S., Liotti, M., Brannan, S. K., McGinnis, S., Mahurin, R. K., Jerabek, P. A., . . Fox, P. T. (1999). Reciprocal limbic-cortical function and negative mood: Converging PET findings in depression and normal sadness. American Journal of Psychiatry, 156, 675-682.

Murphy, F. C., Nimmo-Smith, I., \& Lawrence, A. (2003). Functional neuroanatomy of emotions: A meta-analysis. Cognitive, Affective, \& Behavioral Neuroscience, 3, 207-233.

O'Doherty, J. P. (2004). Reward representations and reward-related learning in the human brain: Insights from neuroimaging. Current Opinion in Neurobiology, 14, 769-776.

Ortony, A., Clore, G. L., \& Collins, A. (1988). The cognitive structure of emotions. Cambridge, United Kingdom: Cambridge University Press.

Ortony, A., \& Turner, T. J. (1990). What's basic about basic emotions? Psychological Review, 97, 315-331.

Phan, K. L., Wager, T., Taylor, S. F., \& Liberzon, I. (2002). Functional neuroanatomy of emotion: A meta-analysis of emotion activation studies in PET and fMRI. NeuroImage, 16, 331-348.

Plutchik, R. (1962). The emotions: Facts, theories, and a new model. New York: Crown.

Plutchik, R. (1980). A general psychoevolutionary theory of emotion. In R. Plutchik \& H. Kellerman (Ed.), Emotion: Theory, research, and experience. Vol. 1: Theories of emotion (pp. 3-31). New York: Academic Press.

Rolls, E. T. (1999). The brain and emotion. New York: Oxford University Press.

Roseman, I. J. (1984). Cognitive determinants of emotion: A structural theory. Review of Personality and Social Psychology, 5, 11-36.

Russell, J. A. (1980). A circumplex model of affect. Journal of Personality and Social Psychology, 39, 1161-1178.

Russell, J. A. (2003). Core affect and the psychological construction of emotion. Psychological Review, 110, 145-172.

Schachter, S., \& Singer, J. E. (1962). Cognitive, social, and physiological determinants of emotional state. Psychological Review, 69, 379399

Scherer, K. R. (1984). On the nature and function of emotion: A component process approach. In K. R. Scherer \& P. Ekman (Eds.), Approaches to emotion (pp. 293-317). Hillsdale, NJ: Erlbaum.

Scherer, K. R. (1993). Studying the emotion-antecedent appraisal process: An expert system approach. Cognition \& Emotion, 7, 325-355.

Schlosberg, H. (1952). The description of facial expressions in terms of two dimensions. Journal of Experimental Psychology, 44, 229-237.

Schultz, W., \& Dickinson, A. (2000). Neuronal coding of prediction errors. Annual Review of Neuroscience, 23, 473-500.

Schwartz, C. E., Wright, C. I., Shin, L. M., Kagan, J., Whalen, P. J., McMullin, K. G., \& Rauch, S. L. (2003). Differential amygdalar response to novel versus newly familiar neutral faces: A functional MRI probe developed for studying inhibited temperament. Biological Psychiatry, 53, 854-862.

Shaver, P., Schwartz, J., Kirson, D., \& O’Connor, C. (1987). Emotion knowledge: Further exploration of a prototype approach. Journal of Personality and Social Psychology, 52, 1061-1086.

Smith, C. A., \& Ellsworth, P. C. (1985). Patterns of cognitive appraisal in emotion. Journal of Personality and Social Psychology, 48, 813838

Storm, C., \& Storm, T. (1987). A taxonomic study of the vocabulary of emotions. Journal of Personality and Social Psychology, 53, 805816

Watson, D., \& Tellegen, A. (1985). Toward a consensual structure of mood. Psychological Bulletin, 98, 219-235.

Whalen, P. J., Rauch, S. L., Etcoff, N. L., McInerney, S. C., Lee, M. B., \& Jenike, M. A. (1998). Masked presentations of emotional facial expressions modulate amygdala activity without explicit knowledge. Journal of Neuroscience, 18, 411-418.

Wilson, F. A. W., \& Rolls, E. T. (1993). The effects of stimulus novelty and familiarity on neuronal activity in the amygdala of monkeys per- 
forming recognition memory tasks. Experimental Brain Research, 93, $367-382$.

Wunderlich, K., Rangel, A., \& O’Doherty, J. P. (2009). Neural computations underlying action-based decision making in the human brain. Proceedings of the National Academy of Sciences USA, 106, 1719917204.

Yik, M. S. M., Russell, J. A., \& Barrett, L. F. (1999). Structure of self-reported current affect: Integration and beyond. Journal of Personality and Social Psychology, 77, 600-619.
Zelazo, P. D., \& Cunningham, W. A. (2007). Executive function: Mechanisms underlying emotion regulation. In J. Gross (Ed.), Handbook of emotion regulation (pp. 135-158). New York: Guilford Press.

Received January 19, 2011 Revision received April 25, 2011 Accepted May 4, 2011

\section{Members of Underrepresented Groups: Reviewers for Journal Manuscripts Wanted}

If you are interested in reviewing manuscripts for APA journals, the APA Publications and Communications Board would like to invite your participation. Manuscript reviewers are vital to the publications process. As a reviewer, you would gain valuable experience in publishing. The $\mathrm{P} \& \mathrm{C}$ Board is particularly interested in encouraging members of underrepresented groups to participate more in this process.

If you are interested in reviewing manuscripts, please write APA Journals at Reviewers@apa.org. Please note the following important points:

- To be selected as a reviewer, you must have published articles in peer-reviewed journals. The experience of publishing provides a reviewer with the basis for preparing a thorough, objective review.

- To be selected, it is critical to be a regular reader of the five to six empirical journals that are most central to the area or journal for which you would like to review. Current knowledge of recently published research provides a reviewer with the knowledge base to evaluate a new submission within the context of existing research.

- To select the appropriate reviewers for each manuscript, the editor needs detailed information. Please include with your letter your vita. In the letter, please identify which APA journal(s) you are interested in, and describe your area of expertise. Be as specific as possible. For example, "social psychology" is not sufficient-you would need to specify "social cognition" or "attitude change" as well.

- Reviewing a manuscript takes time (1-4 hours per manuscript reviewed). If you are selected to review a manuscript, be prepared to invest the necessary time to evaluate the manuscript thoroughly. 